\title{
Trend nella terapia sostitutiva con testosterone negli uomini in età riproduttiva dal 2003 al 2013
}

\author{
Alberto Ferlin 1
}

Pubblicato online: 20 dicembre 2016

(C) Springer International Publishing AG 2016

Commento a:

Trends in testosterone replacement therapy use among reproductive-age US men, 2003-2013.

P.K. Rao, S.L. Boulet, A. Mehta, J. Hotaling, M.L. Eisenberg, S.C. Honig, L. Warner, D.M. Kissin, A.K. Nangia, L.S. Ross.

J Urol (2016) doi:10.1016/j.juro.2016.10.063

La terapia sostitutiva con testosterone (TRT) è incrementata notevolmente negli ultimi decenni, rappresentando uno dei mercati farmaceutici in più grande e veloce sviluppo. Allo stesso tempo, la definizione della sindrome da basso testosterone e la caratterizzazione dei sintomi, segni e comorbidità dell'ipogonadismo maschile, in particolare quello dell'età avanzata, sono stati meglio definiti, così come sono proliferati studi sugli effetti benefici, collaterali e complicanze a lungo termine della TRT. Tuttavia, la maggior parte di questi studi si sono focalizzati sulla popolazione anziana, cioè sui soggetti affetti da late onset hypogonadism. Molto meno è noto riguardo i soggetti più giovani, in età riproduttiva, in cui non solo il trend di utilizzo della TRT non è ben noto, ma anche gli effetti negativi sul potenziale di fertilità e gli effetti a lungo termine sono potenzialmente più importanti.

In questo studio è stato analizzato il trend di utilizzo della TRT negli USA nei soggetti di 18-45 anni tra il 2003 e il 2013, estrapolando i dati dai database assicurativi e dei servizi farmaceutici (più di 5 milioni di soggetti inseriti nel database nel 2013). I dati sono stati confrontati con i soggetti di 56-64 anni ed è stata preso in considerazione anche

$凶$ A. Ferlin

alberto.ferlin@unipd.it

1 Dipartimento di Medicina, UOC Andrologia e Medicina della Riproduzione, Università di Padova, Padova, Italia il numero di soggetti che avevano eseguito un esame del liquido seminale e/o un dosaggio del testosterone meno di un anno prima dall'inizio della TRT.

I risultati evidenziano che tra il 2003 e il 2013 si è verificato un incremento di quattro volte nell'utilizzo della TRT nei soggetti di 18-45 anni (da 29,2 a 118,1 per 10000 soggetti, corrispondente a circa 6000 soggetti nel 2003 e 60000 nel 2013) e di 3 volte nei soggetti di 55-64 anni (da 125,2 a 374,3 per 10000 soggetti, corrispondente a circa 8000 soggetti nel 2003 e più di 63000 nel 2013). Nella popolazione più giovane, la formulazione in gel è incrementata percentualmente dal 2003 al 2008 (66,8 e 75,0\% rispettivamente), ma poi è calata al $44,6 \%$ nel 2013 , superata in quest'ultimo anno dalle formulazioni iniettive che sono incrementate percentualmente dal 2003 al 2013 (20,7 e 56,1\% rispettivamente). Meno del $2 \%$ dei soggetti aveva eseguito un esame del liquido seminale prima dell'inizio della TRT $(1,8 \%$ nel 2004 e 1,2\% nel 2013), mentre la percentuale di soggetti che ha eseguito la determinazione del testosterone prima della TRT è incrementata progressivamente dal 33,2\% nel 2003 al 79,7\% nel 2013.

Questo studio riporta per la prima volta il trend di utilizzo della TRT nei soggetti in età riproduttiva e invita ad alcune riflessioni, quali l'incremento in termini assoluti e lo shift verso le formulazioni iniettive a discapito di quelle topiche. Nonostante l'incremento del dosaggio del testosterone, rimane un $20 \%$ di soggetti che intraprendono la TRT senza alcuna determinazione del testosterone, elemento essenziale per diagnosticare l'ipogonadismo e la necessità di TRT. Analizzando questi numeri, gli effetti negativi sul potenziale di fertilità non sembrano essere adeguatamente considerati $\mathrm{e}$ appare un possibile utilizzo del testosterone non appropriato e/o con indicazioni non approvate. 